\title{
Role of indocyanine green fluorescence imaging on blood flow evaluation of the reconstructed conduit after esophageal cancer surgery
}

\author{
Kazuo Koyanagi* and Soji Ozawa \\ Department of Gastroenterological Surgery, Tokai University School of Medicine, Isehara, Japan
}

Esophagectomy remains a standard curative treatment for localized thoracic esophageal cancer. However, morbidity is a major concern after surgery because of the surgical invasiveness and the complex operative procedures. The anastomosis between the cervical esophagus and the reconstructed conduit is more likely to leak than other gastrointestinal anastomosis and is consequently associated with higher postoperative mortality. Among several factors, such as patient factors, surgical techniques, and tissue factors, that is a high probability that ischemia at the tip of the reconstructed conduit can attribute to the anastomotic leakage. Traditional practice has been to check the pulsation of the marginal artery, the color of the conduit, and/or bleeding from the cutting edge of the bowel. These methods are all subjective and rarely provide a clear demarcation between good blood perfusion area and non-good blood perfusion area of the reconstructed conduit.

Instead, to obtain the objective information of blood perfusion of the reconstructed conduit and to decide the appropriate anastomotic site after esophagectomy, laser Doppler flowmetry emerged as a tissue blood flow assessment system. However, it has not been widely accepted by surgeons due to the lack of reproducibility and no apparent correlation with anastomotic leakage. Near-infrared fluorescence imaging requires a fluorescence-imaging agent that can be excited in the organs at nearinfrared wavelengths by penetrating near-infrared light. The image of the near-infrared fluorescence can be created based on the fluorescence generated by the excited near-infrared fluorescence agent and captured by adapted cameras. Indocyanine green (ICG) is a clinically approved near-infrared fluorescence agent and has been applied as a real-time navigation tool in various surgical fields. ICG is a water-soluble nearinfrared fluorophore, with its immediate or long-term safety confirmed. ICG is known for its maximum absorption around 760-780 nm and binds with plasma proteins immediately resulting in a confinement to the vascular compartment. ICG fluorescence imaging provides high sensitivity and significant contrast because of its low inherent autofluorescence background and high tissue penetration. Recent studies have shown that ICG fluorescence can visualize the blood flow of the gastric conduit in patients undergoing esophagectomy.

We have demonstrated that ICG fluorescence imaging can be used to effectively visualize real-time blood flow of the gastric conduit [1]. We focused on the blood flow speed from the pylorus to the terminal end of the ICG fluorescence stream in the gastric conduit wall. We realized that the ICG fluorescence stream in the gastric conduit wall could be classified into two patterns, a simultaneous group where the stream speed was fast, and a delayed group where the stream speed was slow, then, found that anastomotic leakage was significantly correlated with the flow speed of ICG fluorescence in the gastric conduit wall. Besides us, advanced surgeons have adopted ICG fluorescence imaging during esophageal cancer surgery and reported the usefulness of that system for reducing the risk of anastomotic leakage in recent years. Based on such studies, one systematic review and one meta-analysis assessed the usefulness of ICG fluorescence imaging for prevention of anastomotic leakage after esophagectomy. In the review article by Daele et al. [2] they selected 19 articles and showed that anastomotic leakage occurred significantly lower in the ICG guided group than in the control group. The anastomotic leakage rate in the group whose surgical procedures were changed based on the ICG fluorescence imaging was similar to that of the good perfusion group, and significantly less than the nongood perfusion group. In the study, the anastomotic leakage rate ranged from $0 \%$ to $33 \%$. Only five studies, including our study, attempted to quantify the ICG fluorescence imaging of the gastric conduit. In the meta-analysis article by Ladak et al. [3] they selected 17 articles and showed that anastomotic leakage occurred in $10 \%$ in patients with use of ICG fluorescence imaging. The pooled sensitivity and specificity of ICG for predicting postoperative leak were $0.78(P=0.0889)$ and $0.74(P=0.0116)$, respectively. Six trials compared ICG with an intraoperative intervention to improve perfusion to no ICG. The absolute risk reduction with ICG intervention was significant and was found to be $69 \%$ with low heterogeneity among studies. Both papers include more than 1000 patients and are up-to-date analysis assessing the usefulness of ICG fluorescence imaging as a tool to prevent the anastomotic leakage after esophagectomy. However, 16 papers, about $90 \%$ of selected studies, are duplicated in both systematic review and meta-analysis. As Daele EV mentioned, only a few studies attempted to quantify of ICG fluorescence imaging. Furthermore, to evaluate the usefulness of ICG fluorescence in the gastric conduit, there are several issues to be considered, including the observation site, detection time, and the parameters and methods of ICG fluorescence imaging. Actually, both papers could not mention about any established method to detect and evaluate the ICG fluorescence imaging on the gastric conduit after esophagectomy. In other words, previous reports could demonstrate the usefulness of ICG fluorescence imaging as a tool to prevent the anastomotic leakage after esophagectomy, however, could not show any particular evaluation method of ICG fluorescence imaging yet.

${ }^{\star}$ Correspondence to: Kazuo Koyanagi, 143 Shimokasuya, Isehara, Kanagawa 259-1193, Japan, Tel: +81-463-93-1121, Fax: +81-463-95-6491; E-mail: kkoyanagi@tsc.u-tokai.ac.jp

Received: September 29, 2019; Accepted: October 09, 2019; Published: October 14,2019 
The dawn of ICG fluorescence imaging during esophageal cancer surgery has just begun. Until now, the assay system generally depends on the idea of individual researchers. Several factors, such as gastric mucosal atrophy, gastric ulcer, and prior therapy, may affect the results of ICG fluorescence imaging. In the reconstructed conduit after esophagectomy, it may be very important to differentiate between inflow impairment and outflow impairment of the gastric conduit wall. In addition, it will be important to investigate surgical techniques that can increase the blood flow of the reconstructed conduit, and to locate the exact anastomotic point in which blood perfusion is sufficient for anastomotic healing. ICG fluorescence imaging can be performed repeatedly in a less-invasive, reproducible assessment of the reconstructed conduit blood flow. Therefore, ICG fluorescence imaging can be used for evaluation of blood perfusion in the reconstructed conduit as real-time surgical navigation.

\section{References}

1. Koyanagi K, Ozawa S, Oguma J, Kazuno A, Yamazaki Y, et al. (2016) Blood flow speed of the gastric conduit assessed by indocyanine green fluorescence: New predictive evaluation of anastomotic leakage after esophagectomy. Medicine 95: e4386. [Crossref]

2. Daele EV, Nieuwenhove YV, Ceelen W, Vanhove C, Braeckman BP, et al. (2019) Near-infrared fluorescence guided esophageal reconstructive surgery: A systematic review. World J Gastrointest Oncol 11: 250-263. [Crossref]

3. Ladak F, Dang JT, Switzer N, Mocanu V, Tian C, et al. Indocyanine green for the prevention of anastomotic leaks following esophagectomy: A meta-analysis. Surg Endosc 33: 384-394. [Crossref]

Copyright: @2019 Koyanagi K. This is an open-access article distributed under the terms of the Creative Commons Attribution License, which permits unrestricted use, distribution, and reproduction in any medium, provided the original author and source are credited. 